\title{
Erratum to: Anesthetic Selection and Disease-Free Survival Following Optimal Primary Cytoreductive Surgery for Stage III Epithelial Ovarian Cancer
}

\author{
Kevin M. Elias, MD ${ }^{1,2,3,4,5}$, Stephanie Kang, BS $^{2}$, Xiaoxia Liu, MS $^{2}$, Neil S. Horowitz, MD ${ }^{1,4,5}$, Ross S. Berkowitz, \\ $\mathrm{MD}^{1,4,5}$, and Gyorgy Frendl, MD, PhD, $\mathrm{FCCM}^{2,3,5}$ \\ ${ }^{1}$ Division of Gynecologic Oncology, Department of Obstetrics and Gynecology and Reproductive Biology, Brigham and \\ Women's Hospital, Boston, MA; ${ }^{2}$ Surgical ICU Translational Research Center, Brigham and Women's Hospital, Boston, \\ MA; ${ }^{3}$ Department of Anesthesiology, Perioperative and Pain Medicine, Brigham and Women's Hospital, Boston, MA; \\ ${ }^{4}$ Dana-Farber Cancer Institute, Boston, MA; ${ }^{5}$ Harvard Medical School, Boston, MA
}

ERRATUM TO: ANN SURG ONCOL DOI: 10.1245/S10434-014-4112-9
Table 4 in the original article contains some errors. Following is the corrected table:

TABLE 4 Cox's proportional hazards model of selected parameters and relationship to disease free survival

\begin{tabular}{|c|c|c|c|c|c|}
\hline \multirow{2}{*}{$\begin{array}{l}\text { Parameter } \\
\text { Age }\end{array}$} & \multirow{2}{*}{$\begin{array}{l}\text { Parameter estimate } \\
-0.00718\end{array}$} & \multirow{2}{*}{$\begin{array}{l}\text { Hazard ratio } \\
0.993\end{array}$} & \multicolumn{2}{|c|}{$95 \%$ hazard ratio confidence limits } & \multirow{2}{*}{$\frac{p \text { value }}{0.45}$} \\
\hline & & & 0.974 & 1.012 & \\
\hline BMI & 0.02556 & 1.026 & 0.991 & 1.062 & 0.15 \\
\hline Race & 0.42777 & 1.534 & 0.977 & 2.407 & 0.06 \\
\hline \multicolumn{6}{|l|}{ Anesthesia } \\
\hline General alone & & 1.00 (ref) & & & \\
\hline General and epidural & -0.30690 & 0.736 & 0.491 & 1.103 & 0.14 \\
\hline Carcinosarcoma & 1.46396 & 4.323 & 1.950 & 9.585 & $<0.001$ \\
\hline \multicolumn{6}{|l|}{ Cancer stage } \\
\hline IIIa & -1.05845 & 0.347 & 0.123 & 0.976 & 0.04 \\
\hline IIIb & -0.28471 & 0.752 & 0.388 & 1.457 & 0.40 \\
\hline IIIc & & 1.00 (ref) & & & \\
\hline Estimated blood loss & -0.0001034 & 1.000 & 0.999 & 1.000 & 0.62 \\
\hline $\mathrm{RBC}$ transfusion & 0.52880 & 1.697 & 0.995 & 2.894 & 0.05 \\
\hline Length of case & -0.12754 & 0.880 & 0.690 & 1.123 & 0.30 \\
\hline
\end{tabular}

The online version of the original article can be found under doi: 10 . 1245/s10434-014-4112-9.

(C) Society of Surgical Oncology 2015

Published Online: 29 January 2015

K. M. Elias, MD

e-mail: kelias1@partners.org 
TABLE 4 continued

\begin{tabular}{|c|c|c|c|c|c|}
\hline Parameter & Parameter estimate & Hazard ratio & \multicolumn{2}{|c|}{$95 \%$ hazard ratio confidence limits } & $p$ value \\
\hline Sevoflurane & & 1.00 (ref) & & & \\
\hline Desflurane & -0.57364 & 0.563 & 0.330 & 0.962 & 0.04 \\
\hline \multicolumn{6}{|c|}{ Level of surgical debulking } \\
\hline No residual & & 1.00 (ref) & & & \\
\hline$<5 \mathrm{~mm}$ residual & 0.57596 & 1.779 & 1.166 & 2.713 & 0.01 \\
\hline $5 \mathrm{~mm}-1 \mathrm{~cm}$ residual & 1.12904 & 3.093 & 1.727 & 5.538 & $<0.001$ \\
\hline
\end{tabular}

